\title{
Effectiveness of Azadirachta indica (neem tree) on prevention and treatment of clinical human malaria: A systematic review
}

\author{
Erasto Vitus Mbugi ${ }^{a}$, Alfred Said Sifeb, Mboni Ruzegea ${ }^{a}$, Grace Emmanuel Peter Msoffec, Bestina \\ Danield, Edward Kabyemela $^{a}$, Bruno Sunguya ${ }^{a}$, Edda Tandi Lwoga ${ }^{e}$ \\ Muhimbili University of Health and Allied Sciences, Dar es Salaam, Tanzania \\ bMoshi Co-operative University, Kilimanjaro, Tanzania \\ The University of Dodoma, Dodoma, Tanzania \\ Tanzania Commission for Science and Technology, Dar es Salaam, Tanzania \\ College of Business Education, Dar es Salaam, Tanzania \\ *Correspondence to Erasto Vitus Mbugi (evmbugi@muhas.ac.tz, erastombugi@gmail.com)
}

\begin{abstract}
Introduction: Neem tree parts such as leaves, stem barks, and roots are known to have some medicinal values in both humans and animals. However, the evidence is scattered and vary with populations and regions. This systematic review sought to explore the effectiveness of neem as a therapeutic and prophylactic agent against malaria.

Methodology: The systematic review examined the effectiveness of neem using the pre-registered review protocol and followed the Preferred Reporting Items for Systematic Reviews and Meta-Analyses (PRISMA) checklist. The Population Intervention Comparator Outcome (PICO) question was: "What is the effectiveness of neem (Azadirachta indica) when used as a therapeutic and prophylactic agent for malaria infection?" It included all cross-sectional survey studies, qualitative studies, case-control studies, randomised controlled trials, quasi-experimental studies, and cohort studies with or without comparison groups. Studies that had both children and adult patients who were diagnosed with malaria were included in the survey. Malaria patients using traditional medications other than neem as well as those who did not use neem were excluded from this study. The search for articles, screening, and synthesis were conducted using the Rayyan software.

Results: Out of the total 1089 articles retrieved, only 3 fitted the inclusion criteria, 1 article could not be retrieved. A narrative synthesis was therefore done on 2 final research articles that were retrievable. The pooled evidence shows that Azadirachta indica is effective against malaria. The medicinal effects are more on symptoms and curbing development to clinical disease than ant parasitic effects.

Conclusion: Neem is potential traditional medicine for malaria symptoms' treatment, but evidence on ant parasitic effects is still not conclusive. The study recommends further primary studies to enhance the power of results to further recommend this plant for the prevention of or treatment of malaria symptoms.
\end{abstract}

Keywords: Malaria, Azadirachta indica, symptomatic treatment, Neem, herb

\section{INTRODUCTION}

$\mathrm{N}$ eem is an evergreen tree, cultivated in various parts of the Indian subcontinent. Neem is cultivated in at least 30 countries worldwide, in Asia, Africa as well as Central and South America. Some small-scale plantations are also reportedly successful in Europe and the United States of America. The Latinised name for neem is Azadirachta indica. It is derived from the Persian, it means "the free tree of India". ${ }^{1}$ According to Biswas et al. ${ }^{2}$, the neem tree was described as Azadirachta indica as early as 1830 and its taxonomic position is as follows: Order-Rutales; Suborder: Rutinae; Family: Meliaceae (mahogany family); Subfamily: Melioideae; Tribe: Melieae; Genus: Azadirachta; and Species: indica. 
Azadirachta indica is perhaps one of the most useful traditional medicinal plants to the extent that it is named "the village pharmacy" in some parts of the world. The therapeutic and prophylactic roles of neem cannot be underestimated in high malaria endemic areas. Anecdotal evidence suggests the potential benefit of each of the part of the neem tree; roots, trunk bark, leaves, and flowers, these traditionally have some medicinal value against various diseases. ${ }^{3-5}$ Neem is said to variably relieve patients symptoms of malaria, stomach and intestinal ulcers, skin diseases, teeth and other pains, respiratory disorders, constipation, rheumatism, chronic syphilitic sores and fever. ${ }^{3}$ It is also reported that neem can work as an anticancer and treatment agent for various disease via the regulation of its various biological and physiological pathways. ${ }^{3}$ Consequently, the plant has been variably and widely used for various medicinal purposes with varying praises. Evidence on complete treatment varies and is not conclusive in available documented reports. Tests conducted at the King Institute of Preventive Medicine indicate that the Siddha neem preparation brought down symptoms and speeded up the recovery of patients affected by dengue. ${ }^{6,7}$ In a similar context, the US National Academy of Science had published a report in 1992 entitled "Neem: A tree for solving global problems" which was 10 years later declared by the United Nations as the "Tree of the 21 st century".

Neem is one of the most extensively researched trees of medicinal value. ${ }^{10}$ The chemical investigations on the products of the neem tree have been undertaken since the middle of the twentieth century, and more than 135 compounds have been isolated from its different parts. ${ }^{2}$ Experimental studies in mice using neem leaf and stem bark extracts showed a significant reduction in mouse malaria parasites - the Plasmodium berghei. ${ }^{11}$ Among human beings, neem use resulted in a decline of Plasmodium falciparum culture asexual and the sexual forms by $50 \%$ compared to control cultures. ${ }^{12}$ In addition, neem has insecticidal effects on malaria vectors. ${ }^{13-15}$ Despite the wide range of potential benefits of neem, the ecogeographic and genetic variation of the plant may influence the concentrations of azadirachtin and other contents in the seeds. ${ }^{16}$ The variations may influence the medicinal value and potential use of the plant for various purposes. The half-life of Azadirachtin varies in different situations. For example, formulations can retain over $59 \%$ of the azadirachtin content for about a year when stored at $10-15^{\circ} \mathrm{C}$ in the dark. The same formulations decompose at higher temperatures, in alkaline and strongly acidic media, particularly in the light. ${ }^{17}$ This photosensitivity necessitates the need of carefulness in storage for uses especially when the extract is used as a pesticide. ${ }^{18}$

Neem plant and its parts are variably and safely used in different geographic locations. It is used as a pesticide as well as ovicidal, larvicidal and adulticidal agent in control of Anopheles mosquitoes, the malaria vector. ${ }^{19}$ This systematic review focused on the effect of neem as a therapeutic and prophylactic agent, restricting information on in vivo studies not extractbased neither in vitro, ex-vivo nor experimental studies. Our focus was dictated by the pronounced use of neem for cure of malaria traditionally, the information that could create a base for proposing the use of neem for malaria control at the household level. Apart from informal use of neem for various purposes in different populations, information on neem as a therapeutic and prophylactic agent in its natural state is limited despite the reported neuroprotective effects in experimental cerebral malaria. ${ }^{20}$

In order to consolidate evidence to effect policy and implementation thereof, systematic collection and appraisal of evidence to refine recommendations are essential. During protocol development in preparation for this review, we could not find a systematic review previously conducted to examine the effectiveness of neem on prevention and treatment of malaria in human beings. This systematic review, therefore, aimed to explore the effectiveness of neem as a therapeutic and prophylactic agent for malaria infection.

\section{METHODS}

\section{Study Design}

This systematic review was conducted to address the following Population Intervention Comparator Outcome (PICO) question: "What is the effectiveness of neem when used as therapeutic and prophylactic agent for malaria infection?" The sample included studies with patients who were diagnosed with malaria. Intervention included studies that had analysed the use of Azadirachta indica singly and not in combination with other herbs for malaria treatment and prevention. The main outcome was the effectiveness of Azadirachta indica in enabling patients to recover from disease after use and impact on prevention, control and treatment of the disease.

The protocol for this study was registered at the PROSPERO database - an international prospective register of systematic reviews. This is an open access online database of systematic review protocols on health-related topics where researchers prospectively 
register their reviews. The study was given a registration number CRD42018097453. We followed the PRISMA criteria and checklist to conduct this review.

\section{Selection of Studies and Databases}

We included all cross-sectional survey studies, qualitative studies, case-control studies, randomised controlled trials, quasi-experimental studies, and cohort studies with or without comparison groups. We excluded potential review articles, opinions, and editorials, even if addressing some of the PICO items. We conducted evidence search in medical databases. The databases included PubMed, The Cochrane Library, EBSCO, CINAHL, HINARI, SCOPUS and Web of Science via Hinari, Popline, lilacs, clinical trial registry, Eric, Science Direct. Moreover, we conducted a grey literature search from respective databases (Appendix 1) and conducted a hand search from relevant articles in the subject matter. We also searched Google scholar to augment results from other databases. Relevant literature and published reports were retrieved from other websites and organizations. We searched literature published in English only without restricting time period.

\section{Inclusion and Exclusion Criteria}

For participants or population, we included studies that had both children and adult patients who were diagnosed with malaria. We excluded studies in which malaria patients had used other traditional medications other than neem as well as those who had not used neem. We further assessed societal perception and attitude on the use of neem as a medicinal plant to control malaria in endemic areas. Additional outcomes included: the improved quality of health following the use of the product as medication, reduced visits to hospital/malaria clinics due to malaria bouts, improved quality in designing malaria studies, increased level of awareness about the usefulness of Azadirachta indica in treating malaria, costeffectiveness compared to standard drugs, and reported side effect. The search terms were developed by information scientists in the team, as attached in Appendix 1.

\section{Data Extraction and Management}

3 reviewers (EVM, MR, and ETL) conducted the search and 2 reviewers (EVM and ETL) initially screened the studies by titles and abstracts only for possible inclusion. EVM and ETL conducted the full-text screening. Agreement on the inclusion of all studies was reached via consensus. Rayyan software was used to conduct the screening. Data extraction was carried out under the guidance of the PRISMA checklist by Moher et al. ${ }^{21,22}$ EVM and ETL independently extracted data from included studies using a standardised data extraction form. Data were entered into an excel sheet by EVM and checked by ETL.

\section{Search Result}

The searches produced a total of 1,089 references, and after de-duplication, we ended up with 825 references (Figure 1). We excluded a total of 772 references basing on their title and abstract. A total of 53 ethnobotanical studies on the use of medicinal plants for the treatment of diseases in humans were retrieved from PubMed as per methods section. 50 publications were excluded from this review because either they dealt with the use of medicinal plants in the treatment of other human infections other than malaria or used other medicinal plants to treat malaria as shown in Figure 1 .

\section{Data Analysis}

The risk of bias of the included studies was assessed by using the quality of included articles through the scale for quantitative and qualitative studies developed by Kmet et al. ${ }^{23}$ The tool for the quantitative studies has 14 items, which can be scored based on the degree to which the specific criteria were met ("yes" = 2, "partial" = 1, "no" $=0$ ). The items that were not applicable to a particular study were scored as " $\mathrm{n} / \mathrm{a}$ ". ${ }^{23}$ That means they were not included in the summary score. The tool for qualitative studies had 10 items, and the scores can be calculated in a similar fashion as for quantitative studies. The "not applicable" option is not allowed for quantitative studies. Therefore, the "summary score for each article was calculated by summing the total scores obtained across the 10 items and dividing by 20 (the total possible scores"). ${ }^{23} 2$ raters (EVM \& ETL) independently performed the critical appraisal and then resolved differences.

\section{Risk of Bias}

The risk of bias was assessed for the two quantitative studies that were selected. The overall scores (Table 1 ) assigned by both reviewers was 0.5 . Both reviewers assigned the same overall score to 2 studies.

We expected to carry out a meta-analysis and therefore to deploy and even subgroup analysis by age, geographical location as well as other demographic characteristics. However, owing to fewer retrieved studies, heterogeneity among the studies, and differences in measurements of the outcome of interest, we could only conduct narrative synthesis. 


\section{RESULTS}

Effectiveness of Azadirachta Indica as a Therapeutic and Prophylactic Agent for Malaria Infection

Evidence from the 2 pooled studies suggests the potential effects of neem as a medicinal plant (Table 1). The first article $^{24}$ reported the ethno pharmacological information about the uses of neem, particularly the leaves, besides the insecticidal one, on the treatment of malaria. Information gathered indicated that treatment is based on the parts of the plant used and the way the plant is used. Identified plant specimens were collected and made into a herbarium voucher. The study considered the high variability and complex chemical constituent of neem, thus used a High-Performance Thin Layer Chromatography (HPTLC) analysis on neem leaves coming from both the Indonesian island of Bali and the Indian subcontinent.

This study produced data on the medical use of traditional preparations from leaves of neem that displayed a wide spectrum of applications. It was noted that in the Indian Subcontinent, neem leaves are used to treat a variety of conditions including dental and gastrointestinal disorders, malaria fevers, skin diseases and as an insect repellent. In the Balinese, neem leaves

\section{FIGURE 1: Flow chart diagram: Publications screening process to get relevant articles that qualified for qualitative synthesis and quality assessment}

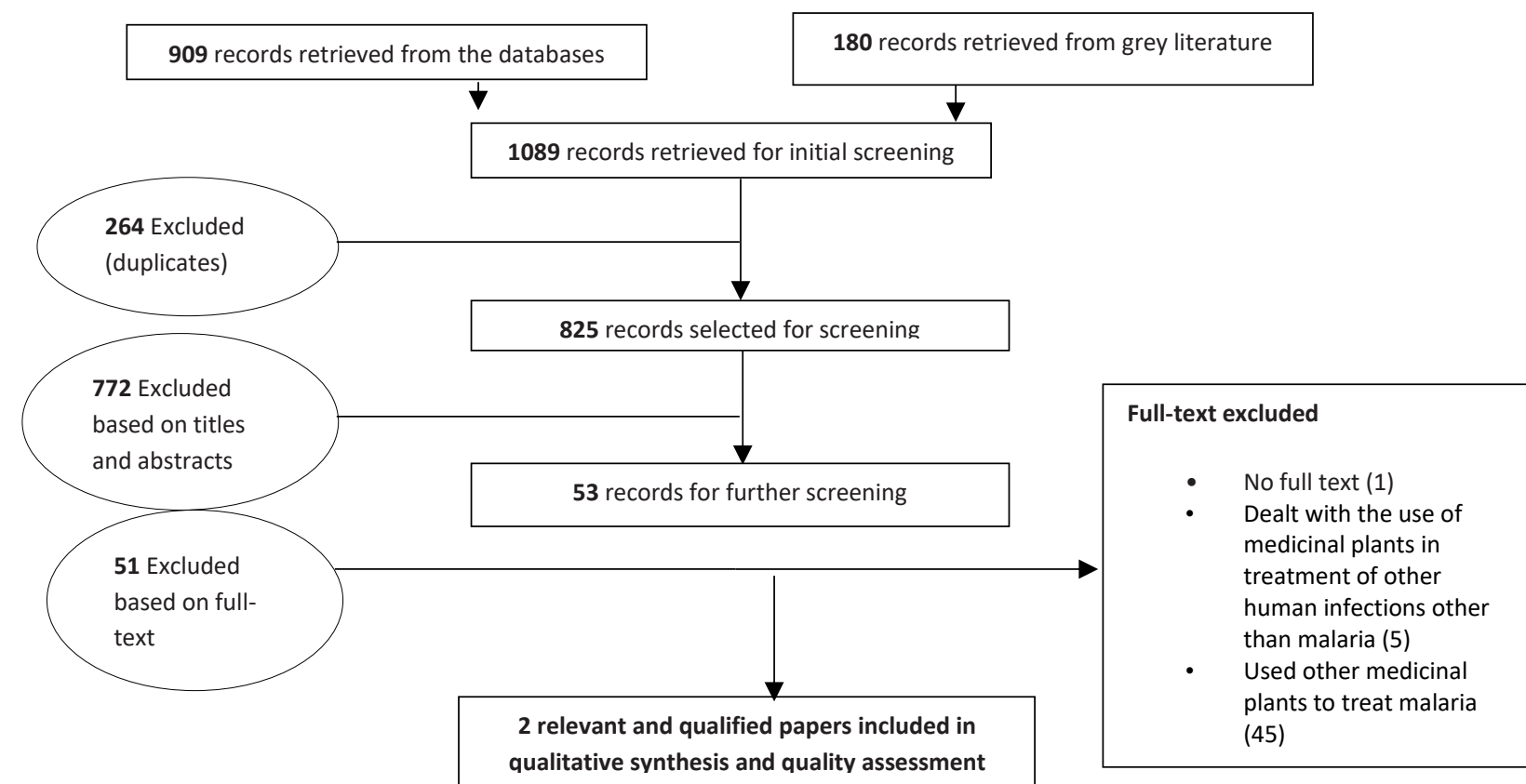




\begin{tabular}{|c|c|c|c|c|}
\hline Criteria & Iwalewa et al (1999) & & Sujarwo et al (2016) & \\
\hline Objective & & 1 & & 0 \\
\hline Design & & 1 & & 0 \\
\hline $\begin{array}{l}\text { Subject/comparison group selection or source } \\
\text { of information/input variables }\end{array}$ & & 1 & & 1 \\
\hline Subject characteristics & & 1 & & 1 \\
\hline Interventional and random allocation & & 1 & $\mathrm{~N} / \mathrm{A}$ & \\
\hline Interventional and blinding of investigators & N/A & & $\mathrm{N} / \mathrm{A}$ & \\
\hline Interventional and blinding of subjects & $\mathrm{N} / \mathrm{A}$ & & N/A & \\
\hline Outcome and (if applicable) exposure measure & & 1 & & 1 \\
\hline Sample size & & 0 & & 2 \\
\hline Analytic methods & & 1 & & 1 \\
\hline $\begin{array}{l}\text { An estimate of variance is reported for the } \\
\text { main results }\end{array}$ & $\mathrm{N} / \mathrm{A}$ & & N/A & \\
\hline Controlled for confounding & $\mathrm{N} / \mathrm{A}$ & & $\mathrm{N} / \mathrm{A}$ & \\
\hline Results reported in sufficient detail & & 1 & & 1 \\
\hline Conclusions supported by the results & & 2 & & 2 \\
\hline Average score & & 0.5 & & 0.5 \\
\hline
\end{tabular}

were used mainly as ant diuretic and anti-diabetes, but also for headache, heartburn, and stimulation of appetite. The authors suggest that the differences in utilisation are not related to chemical differences and other constituents other than limonoids and recommend that further investigations should be considered focusing on its observed multipurpose activity. Informants had good knowledge of neem use in the treatment of diseases with good attitude and practice in the use of neem and other traditional medicines for the treatment of malaria. The study concluded by revealing neem leaves to be believably useful in the treatment of diabetes in both Balinese and Indian communities. The study however had a reservation that limonoids cannot be considered the only factor responsible for digestive properties and recommended further research to validate this report by carrying out enzymatic tests and the identification of active constituents.

Similarly, the second article $^{25}$ explored the contributory pharmacological effects of Azadirachta indica leaf in the treatment of malaria with the aim of assessing the effect of crude extracts on various signs and symptoms of malaria infection in vivo and in vitro. The results showed that the methanolic and diethyl ether extracts have significant antipyretic, analgesic, anti-inflammatory and anti-aggregatory activities, while the chloroform extract did not show appreciable effect. The effects of crude extracts compared favourably with chloroquine in pyrexia, indomethacin in platelet aggregation and Acetylsalicylic Acid (ASA) in analgesia and inflammatory experimental models. The study concluded that the pharmacological effects of these extracts might explain the effectiveness of Azadirachta indica leaf in malaria therapy traditionally.

\section{Screening of articles for qualitative synthesis and quality assessment}

In the screening of retrieved articles for qualitative synthesis and quality assessment (Figure 1), 1 $(1.96 \%)$ out of 51 records of publications was excluded as we could not find its full text. 5 (9.80\%) articles comprised of medicinal plants other than Azadirachta indica used for the treatment of other human infections than malaria. $45(88.24 \%)$ articles comprised of other ethno botanical medicinal plants than Azadirachta indica that were used to treat malaria. This observation indicates that apart from the believed use of Azadirachta indica in the treatment of malaria and other infections, there are several other medicinal plants variably used as medicines to treat malaria and other infections. This necessitates further investigation that may include extraction of ingredients from each plant species of interest and testing for its effectiveness to treat malaria and other infections. Most studies on medicinal plants and infection are regional with more studies coming from the Indian sub-continent, particularly India, Pakistan 
and Indonesia. In Africa, most of the studies relating Azadirachta indica and other medicinal plants come from West African countries, namely Nigeria, Benin, Burkina Faso, and Ghana to mention a few. In Africa, south of Sahara, most of the studies seem to be concentrated in South Africa, with Kenya and Uganda dominating for the East African region. It is however worth noting that despite the limited number of reported studies on Azadirachta indica and other medicinal plants, regional informal use of these plants as medicines for variable medicinal values cannot be underestimated considering the variable cultures and traditions.

\section{Pre-Clinical Studies}

In the screening process, it was noted that most of the research on medicinal plants believed to be useful in treating malaria and other infections had been tried in vitro or in mice/rodents. The results from these trials provide clues on the role of these medicinal plants in human host. We look forward to seeing the next step in this research where the real impacts will be observed in humans. Only 2 publications working on Azadirachta indica and malaria were identified. This number is too small, however, it could not mean that the plant and its products are useless. This could be because most treatments are conducted by traditional healers or by individuals in their homes, thus, such treatments cannot be easily reported due to remoteness. This contributes to the limited information out of many in use.

Despite the novelty of this study and its potential for policy and guidance, evidence gathered should be discussed in light of the following limitations: There is no enough empirical research done around the neem plant. In this systematic review study, only 2 studies out of 53 recorded for further screening met the inclusion criteria and qualified for further analysis. This shows that the use of Azadirachta indica to treat diseases is an area worth further research in order to publicise the therapeutical effect of the neem plant for positive treatment outcome of diseases. Despite the rare publicity of neem as a therapeutic or prophylactic agent, various parts of the plant are informally and variably used in different societies to alleviate individuals from a diverse of disease conditions.

\section{DISCUSSION}

Azadirachta indica is a medicinal plant commonly used to treat various diseases in traditional medicine. It has also been knowingly or unknowingly used prophylactically or as a treatment for malaria in regions where the disease is endemic. Azadirachta indica has shown a wide range of pharmacological activities in traditional medicine, particularly the neem leaf and other non-wood parts of the plant.5,26,27 This systematic review explored the effectiveness of Azadirachta indica in the treatment of malaria infection as a therapeutic or a prophylactic agent. The evidence collected was appraised to be of moderate quality, nevertheless showed that neem has potential to address malaria symptoms though not complete remission. In vitro studies involving the potential roles of diverse bioactive compounds from Azadirachta indica, mainly limonoids including nimbolide, azadarachtin, and gedunin are involved in modulation of multiple cells signalling pathways. ${ }^{28}$ The proposed multiple cellular and molecular mechanisms of bioactive compounds include free radical scavenging, DNA repair, cell cycle alteration, programmed cell death (apoptosis) and autophagy. In addition, the bioactive materials are said to be involved in immune surveillance, anti-inflammatory, anti-angiogenic, antiinvasive and anti-metastatic activities as well as modulation of several dysregulated oncogenic signalling pathways. ${ }^{29}$ The economic importance of neem, however, extends to its evidence-based additional diverse complementary and safe use as mosquitocide..$^{19,30}$

The ethno botanical uses of neem are historical where it has variably and believably been used to alleviate individuals from common diseases such as diabetes mellitus, malaria, various skin conditions and other symptoms such as fever and headaches. ${ }^{24,31,32}$ Reports also show that neem leaf and stem bark extracts have ant malarial effects on Plasmodium berghei infected mice with a significant reduction in parasitaemia speculating its potential for malaria treatment. ${ }^{11}$ Our review on the therapeutic effects indicated that Neem has high potential as antipyretic, analgesic, antiinflammatory as well as anti-platelet aggregation. ${ }^{25}$ Although the antipyretic effects of Azadirachta indica seem to be short-lived, its use and effectiveness can possibly be heightened by complementing other ethno botanical medicinal plants if available or modern drugs such as paracetamol where necessary. The effect however, seems to be symptom-based than curative by relieving patients from symptoms associated with the disease rather than acting on the malaria parasites. In this context, while the effects of Azadirachta indica is traditionally considered therapeutic against malaria, it is in fact, prophylactic in the sense that when used it may reduce the possibility of patient's progression to or persistence of clinical signs resulting from infection by the malaria parasites. 
In this systematic review, the 2 studies $^{24,25}$ variably explored the effectiveness of Azadirachta indica as medicinal plant against malaria but looking at different angles and different approaches. While the Sujarwo et al. study ${ }^{24}$ focused on ethno pharmacological information on uses of neem, in particular the leaves, besides the insecticidal one considering the historical background of their uses, the Lwalewa et al. study ${ }^{25}$ had an interest on the in vivo and in vitro effects of the plant extracts as antipyretic, analgesic, antiinflammatory and anti-aggregatory activities against malaria disease. The plant medicinal value ranges from fruits to roots. ${ }^{24}$ The plant seemed to have variable uses such as to treat dental and gastrointestinal disorders, malaria fevers, skin diseases, as an insect repellent, as a diuretic, and treatment of diabetes, headache, heartburn and stimulating appetite. The antipyretic, analgesic, anti-inflammatory and anti-aggregatory activities were common findings ${ }^{25}$ despite the different methods of testing the effectiveness of the plant leat extracts on malaria. Hence, both studies reveal that Azadirachta indica is effective in the treatment of malaria and its effectiveness to be specifically from the use of leaves or leaf extracts of the plant. The role of Azadirachta indica in the prevention and treatment of diseases is via the regulation of various biological and physiological pathways. ${ }^{3}$

\section{CONCLUSION}

In conclusion, this systematic review shows that although Azadirachta indica can have variable believed medicinal applications such as relieving patients from symptoms of a diverse number of diseases, it is effective against malaria. However, its effects are more on the symptoms and development to clinical disease than acting on the parasite itself to highlight the proposed regulation role on various biological and physiological pathways. Given the historical and traditional belief on the use of Azadirachta indica in the treatment of various diseases, this study recommends further investigations to get more complementary value of the plant in alleviating floods of disease calamities in developing world, especially in malaria-endemic regions. In addition, further investigations on other traditional plants for their values in treating malaria and other infections should also be advocated.

\section{Limitations}

Restricted access to articles in some databases such as Web of Science and Embase because they are not accessible in our region.

\section{REFERENCES}

1. V.S. Kumar and V. Navaratnam, "Neem (Azadirachta indica): Prehistory to contemporary medicinal uses to humankind," Asian Pacific Journal of Tropical Biomedicine, 3 (2013), 50514.

2. K. Biswas, I. Chattopadhyay, R.K. Banerjee and U. Bandyopadhyay, "Biological activities and medicinal properties of neem (Azadirachta indica)," Current Science, 82 (2002), 1336-45.

3. M.A. Alzohairy, "Therapeutics Role of Azadirachta indica (Neem) and Their Active Constituents in Diseases Prevention and Treatment," Evidence-based complementary and alternative medicine : eCAM, 2016 (2016), 7382506-06.

4. G. Nahak and R.K. Sahu, "Bioefficacy of Leaf Extract of Neem (Azadirachta indica A. Juss) on Growth Parameters, Wilt and Leafspot Diseases of Brinjal " Research Journal of Medicinal Plants, 8 (2014), 269-76.

5. R. Subapriya and S. Nagini, "Medicinal Properties of Neem Leaves: A Review, " Current Medicinal Chemistry - Anti-Cancer Agents, 5 (2005), 149-56.

6. P. Narayan, "Neem-papaya juice passes dengue test. The Times of India.," (2012).

7. K. Singh, M. Zeeshan, V.A. Ansari, Z. Ahmad, P. Bagga and P. Shakya, "Prevention and control of dengue by herbal remedies," J Chem Pharm Res, 8 (2016), 708-13.

8. "National Academy of Science. Washington, DC: NAS; 1992. Neem, a tree for solving global problems."

9. UNEP, "United Nations Environment Programme. Neem: The UN's tree of the 21st Century. Nairobi: United Nations Environment Programme; [Online] Available from: http://www.unep.org/wed/tree-a-day/neem.asp," (2012).

10. B. Anyaehie, "Medicinal properties of fractionated acetone/water neem (Azadirachta indica) leaf extract from Nigeria: a review," Nigerian Journal of Physiological Sciences: Official Publication of the Physiological Society of Nigeria, 24 (2009), 157-9.

11. B.C. Akin-Osanaiye, A.J. Nok, S. Ibrahim, H.M. Inuwa, E. Onyike and E. Haruna, "Antimalarial effect of Neem leaf and Neem stem bark extracts on plasmodium berghei infected in the pathology and treatment of malaria," International Journal of Research in Biochemistry and Biophysics, 3 (2013), 7-14.

12. I. Udeinya, E. Shu, I. Quakyi and F. Ajayi, "An Antimalarial Neem Leaf Extract has Both Schizonticidal and Gametocytocidal Activities," American Journal of Therapeutics, 15 (2008), 108-10.

13. G. Benelli, A. Canale, C. Toniolo, A. Higuchi, K. Murugan, R. Pavela and M. Nicoletti, "Neem (Azadirachta indica): towards the ideal insecticide?," Natural Product Research, 31 (2017), 369-86.

14. M.S. Mulla and T. Su, "Activity and biological effects of neem products against arthropods of medical and veterinary importance," J Am Mosq Control Assoc, 15 (1999), 133-52.

15. S.S. Nathan, K. Kalaivani and K. Murugan, "Effects of neem limonoids on the malaria vector Anopheles stephensi Liston (Diptera: Culicidae)," Acta Trop, 96 (2005), 47-55.

16. J.R. Chamberlain, F.J. Childs and P.J.C. Harris, Improvement of neem (Azadirachta indica) and its potential benefits to poor farmers in developing countries (2000).

17. I. Ujváry and R. Krieger, Chapter 3 - Pest Control Agents from Natural Products In Hayes' Handbook of Pesticide Toxicology (Third Edition), (New York: Academic Press, 2010), 119-229.

18. S. Johnson, P. Dureja and S. Dhingra, "Photostabilizers for azadirachtin-A (a neem-based pesticide), " J Environ Sci Health B, 38 (2003), 451-62.

19. B. Chandramohan, K. Murugan, P. Madhiyazhagan, K. 
Kovendan, P.M. Kumar, C. Panneerselvam, D. Dinesh, J. Subramaniam, R. Rajaganesh, M. Nicoletti, A. Canale and G. Benelli, "Neem by-products in the fight against mosquitoborne diseases: Biotoxicity of neem cake fractions towards the rural malaria vector Anopheles culicifacies (Diptera: Culicidae)," Asian Pacific Journal of Tropical Biomedicine, 6 (2016), 472-76.

20. M. Farahna, S. Bedri, S. Khalid, M. Idris, C.R. Pillai and E.A. Khalil, "Anti-plasmodial effects of Azadirachta indica in experimental cerebral malaria: Apoptosis of cerebellar Purkinje cells of mice as a marker," North American Journal of Medical Sciences, 2 (2020), 518-25.

21. D. Moher, A. Liberati, J. Tetzlaff and D.G. Altman, "Preferred reporting items for systematic reviews and meta-analyses: the PRISMA statement," BMJ, 339 (2009), b2535.

22. D. Moher, L. Shamseer, M. Clarke, D. Ghersi, A. Liberati, M Petticrew, P. Shekelle, L.A. Stewart and P.-P. Group, "Preferred reporting items for systematic review and meta-analysis protocols (PRISMA-P) 2015 statement," Systematic Reviews, 4 (2015), 1 .

23. L.M. Kmet, R.C. Lee and L.S. Cook, Standard quality assessment criteria for evaluating primary research papers from a variety of fields. Edmonton: Alberta Heritage Foundation for Medical Research (AHFMR). AHFMR - HTA Initiative \#13 (2004).

24. W. Sujarwo, A.P. Keim, G. Caneva, C. Toniolo and M. Nicoletti, "Ethnobotanical uses of neem (Azadirachta indica A.Juss.; Meliaceae) leaves in Bali (Indonesia) and the Indian subcontinent in relation with historical background and phytochemical properties," J Ethnopharmacol, 189 (2016), 186-93.

25. E.O. Iwalewa, K. Mohammed and O.O. Omotola, "Contributory Pharmacological Effects of Azadirachta indica Leaf in the Treatment of Malaria," Nig J Nat Prod Med, 3 (1999), 42-46.

26. K. Girish and B.S. Shankara, "Neem - A Green Treasure," Electronic Journal of Biology, 4 (2008), 102-11.

27. I.P. Ogbuewu, Y.U. Odoemenam, H.O. Obikaonu, M.N. Opara, O.O. Emenalom, M.C. Uchegbu, I.C. Okoli, B.O. Esonu and M.U. Iloeje, "The Growing Importance of Neem (Azadirachta indica A. Juss) in Agriculture, Industry, Medicine and Environment: A Review " Research Journal of Medicinal Plants, 5 (2011), 230-45.

28. S.C. Gupta, S. Prasad, A.K. Tyagi, A.B. Kunnumakkara and
B.B. Aggarwal, "Neem (Azadirachta indica): An indian traditional panacea with modern molecular basis," Phytomedicine, 15 (2017), 14-20.

29. S. Patel, K. Venkata, P. Bhattacharyya, G. Sethi and A. Bishayee, "Potential of neem (Azadirachta indica L.) for prevention and treatment of oncologic diseases," Seminars in Cancer Biology, 40-41 (2016).

30. M. Nicoletti, K. Murugan, A. Canale and G. Benelli, "NeemBorne Molecules as Eco-Friendly Control Tools Against Mosquito Vectors of Economic Importance," Current Organic $\begin{array}{llll}\text { Chemistry, } & 20 & \text { (2016), }\end{array}$ $10.2174 / 1385272820666160218233923$.

31. W. Kooti, M. Farokhipour, Z. Asadzadeh, D. Ashtary-Larky and M. Asadi-Samani, "The role of medicinal plants in the treatment of diabetes: a systematic review," Electronic physician, 8 (2016), 1832-42.

32. A.L. Wilson, V. Chen-Hussey, J.G. Logan and S.W. Lindsay, "Are topical insect repellents effective against malaria in endemic populations? A systematic review and meta-analysis," Malaria journal, 13 (2014), 446-46.

\section{Peer Reviewed \\ Competing Interests: None declared. \\ Received: 12/30/2020; Accepted: 4/7/2021}

Cite this article as: Mbugi EV, Sife AS, Ruzegea M, Msoffe GEP, Daniel B, Kabyemela E, Sunguya B, Lwoga ET. Azadirachta indica in prophylaxis and treatment of malaria. E Afr Sci. 2021;3(1):34-43. http://doi.org/10.24248/EASci-D-20-00017

(C) Mbugi EV et al. This is an open-access a rticle distributed under the terms of the Creative Commons Attribution License, which permits unrestricted use, distribution, and reproduction in any medium, provided the original author and source are properly cited. To view a copy of the license, visit http://creativecommons.org/licens- es/by/4.0/. When linking to this article, please use the following permanent link: http://doi.org/10.24248/EASci-D-20-00017

\title{
APPENDIX 1
}

\section{Pubmed}

\author{
\#6 Search (\#5 NOT \#4) \\ \#5 Search (\#1 AND \#2 AND \#3) \\ \#4 Search ((animals[mesh] NOT humans[mesh])) \\ Search (( (prevention and control [Subheading] OR "primary prevention" OR "secondary prevention" OR \\ \#3 "tertiary prevention" OR prophylaxis OR "preventive therapy" OR "preventive measures" OR prevention OR \\ control OR therapy OR treatment OR "disease management" OR therapeutics ))) \\ Search (((Malaria[mesh] OR "Remittent Fever" OR "Fever Remittent" OR paludism OR "plasmodium \\ infection*" OR "infection* plasmodium" OR "Marsh Fever" OR "Fever Marsh" ))) \\ Search (((azadirachta[mesh] OR azadirachta* OR "Neem tree*" OR "tree* Neem" OR "azadirachta indica*" OR \\ \#1 "indica* azadirachta" OR "melia azadirachta*" OR "azadirachtas melia" )))

\section{CINAHL}


S5

S4

S3

S2

S 1
MH animals NOT MH humans

S1 AND S2 AND S3

MH malaria OR "Remittent Fever" OR "Fever Remittent" OR paludism OR "plasmodium infection*" OR "infection* plasmodium" OR "Marsh Fever" OR "Fever Marsh"

$\mathrm{MH}$ "Preventive Health Care+" OR "primary prevention" OR "secondary prevention" OR "tertiary prevention" OR prophylaxis OR "preventive therapy" OR "preventive measures" OR prevention OR control OR therapy OR treatment OR "disease management" OR therapeutics

MH azadirachta OR azadirachta* OR "Neem tree*" OR "tree* Neem" OR "azadirachta indica*" OR "indica* azadirachta" OR "melia azadirachta*" OR "azadirachtas melia"

\section{SCOPUS through Research4Life programmes}

ALL ( azadirachta* OR "Neem tree*" OR "tree* Neem" OR "azadirachta indica*" OR "indica* azadirachta" OR "melia azadirachta*" OR "azadirachtas melia" ) AND ALL ( malaria OR "Remittent Fever" OR "Fever Remittent" OR paludism OR "plasmodium infection*" OR "infection* plasmodium" OR "Marsh Fever" OR "Fever Marsh" ) AND ALL ( "primary prevention" OR "secondary prevention" OR "tertiary prevention" OR prophylaxis OR "preventive therapy" OR "preventive measures" OR prevention OR control OR therapy OR treatment OR "disease management" OR therapeutics)

\section{Popline}

(azadirachta OR azadirachta* OR "Neem tree*" OR "tree* Neem" OR "azadirachta indica*" OR "indica* azadirachta" OR "melia azadirachta*" OR "azadirachtas melia") AND ( malaria OR "Remittent Fever" OR "Fever Remittent" OR paludism OR "plasmodium infection*" OR "infection* plasmodium" OR "Marsh Fever" OR "Fever Marsh") AND ( "primary prevention" OR "secondary prevention" OR "tertiary prevention" OR prophylaxis OR "preventive therapy" OR "preventive measures" OR prevention OR control OR therapy OR treatment OR "disease management" OR therapeutics)

\section{Web of Science through Research4life programmes}

TS $=$ (azadirachta* OR "Neem tree*" OR "tree* Neem" OR "azadirachta indica*" OR "indica* azadirachta" OR "melia azadirachta*" OR "azadirachtas melia" ) AND TS=( malaria OR "Remittent Fever" OR "Fever Remittent" OR paludism OR "plasmodium infection*" OR "infection* plasmodium" OR "Marsh Fever" OR "Fever Marsh" ) AND TS=( "primary prevention" OR "secondary prevention" OR "tertiary prevention" OR prophylaxis OR "preventive therapy" OR "preventive measures" OR prevention OR control OR therapy OR treatment OR "disease management" OR therapeutics)

\section{ERIC}

(azadirachta OR azadirachta* OR "Neem tree*") AND (malaria OR "Remittent Fever" OR "plasmodium infection*") AND (prevention OR control)

\section{ScienceDirect}

All fields

(azadirachta OR azadirachta* OR "Neem tree*") AND (malaria OR "Remittent Fever" OR "plasmodium infection*")

limit to Title, abstract or (azadirachta OR azadirachta* OR "Neem tree*") AND (malaria OR "Remittent Fever" OR keywords

\section{Lilacs}

(azadirachta OR azadirachta* OR "Neem tree*" OR "tree* Neem" OR "azadirachta indica*" OR "indica* azadirachta" OR "melia azadirachta*" OR "azadirachtas melia" ) AND ( malaria OR "Remittent Fever" OR "Fever Remittent" OR paludism OR "plasmodium infection*" OR "infection* plasmodium" OR "Marsh Fever" OR "Fever Marsh") AND ( "primary prevention" OR "secondary prevention" OR "tertiary prevention" OR prophylaxis OR "preventive therapy" OR "preventive measures" OR prevention OR control OR therapy OR treatment OR "disease management" OR therapeutics) 


\section{Google scholar}

intitle:(azadirachta OR "Neem tree*") AND intitle:(malaria OR "Remittent Fever" OR "plasmodium infection*") 